\title{
First Dark Matter Search Results from the XENON1T Experiment
}

E. Aprile, ${ }^{1}$ J. Aalbers, ${ }^{2,}{ }^{*}$ F. Agostini,${ }^{3,4}$ M. Alfonsi, ${ }^{5}$ F. D. Amaro, ${ }^{6}$ M. Anthony, ${ }^{1}$ F. Arneodo, ${ }^{7}$ P. Barrow,${ }^{8}$ L. Baudis, ${ }^{8}$ B. Bauermeister, ${ }^{9}$ M. L. Benabderrahmane, ${ }^{7}$ T. Berger, ${ }^{10}$ P. A. Breur, ${ }^{2}$ A. Brown, ${ }^{2}$ A. Brown, ${ }^{8}$ E. Brown, ${ }^{10}$ S. Bruenner,${ }^{11}$ G. Bruno, ${ }^{3}$ R. Budnik, ${ }^{12}$ L. Bütikofer, ${ }^{13, \dagger}$ J. Calvén, ${ }^{9}$ J. M. R. Cardoso,${ }^{6}$ M. Cervantes, ${ }^{14}$ D. Cichon, ${ }^{11}$ D. Coderre, ${ }^{13}$ A. P. Colijn, ${ }^{2}$ J. Conrad,${ }^{9}$ J. P. Cussonneau, ${ }^{15}$ M. P. Decowski, ${ }^{2}$ P. de Perio, ${ }^{1}$ P. Di Gangi, ${ }^{4}$ A. Di Giovanni, ${ }^{7}$ S. Diglio, ${ }^{15}$ G. Eurin, ${ }^{11}$ J. Fei, ${ }^{16}$ A. D. Ferella, ${ }^{9}$ A. Fieguth, ${ }^{17}$ W. Fulgione ${ }^{3,18}$ A. Gallo Rosso, ${ }^{3}$ M. Galloway, ${ }^{8}$ F. Gao, ${ }^{1}$ M. Garbini, ${ }^{4}$ R. Gardner, ${ }^{19}$ C. Geis,${ }^{5}$ L. W. Goetzke, ${ }^{1}$ L. Grandi, ${ }^{19}$ Z. Greene, ${ }^{1}$ C. Grignon, ${ }^{5}$ C. Hasterok, ${ }^{11}$ E. Hogenbirk, ${ }^{2}$ J. Howlett, ${ }^{1}$ R. Itay, ${ }^{12}$ B. Kaminsky, ${ }^{13, \dagger}$ S. Kazama, ${ }^{8}$ G. Kessler, ${ }^{8}$ A. Kish, ${ }^{8}$ H. Landsman, ${ }^{12}$ R. F. Lang, ${ }^{14}$ D. Lellouch, ${ }^{12}$ L. Levinson, ${ }^{12}$ Q. Lin, ${ }^{1}$ S. Lindemann, ${ }^{11,13}$ M. Lindner, ${ }^{11}$ F. Lombardi, ${ }^{16}$ J. A. M. Lopes, ${ }^{6, *}$ A. Manfredini, ${ }^{12}$ I. Mariş, ${ }^{7}$ T. Marrodán Undagoitia, ${ }^{11}$ J. Masbou, ${ }^{15}$ F. V. Massoli, ${ }^{4}$ D. Masson, ${ }^{14}$ D. Mayani,${ }^{8}$ M. Messina, ${ }^{1}$ K. Micheneau, ${ }^{15}$ A. Molinario, ${ }^{3}$ K. Morå, ${ }^{9}$ M. Murra,${ }^{17}$ J. Naganoma,${ }^{20}$ K. Ni, ${ }^{16}$ U. Oberlack,${ }^{5}$ P. Pakarha,${ }^{8}$ B. Pelssers, ${ }^{9}$ R. Persiani, ${ }^{15}$ F. Piastra, ${ }^{8}$ J. Pienaar, ${ }^{14}$ V. Pizzella, ${ }^{11}$ M.-C. Piro, ${ }^{10}$ G. Plante,${ }^{1, \S}$ N. Priel,${ }^{12}$ L. Rauch, ${ }^{11}$ S. Reichard, ${ }^{8,14}$ C. Reuter, ${ }^{14}$ B. Riedel, ${ }^{19}$ A. Rizzo, ${ }^{1}$ S. Rosendahl,${ }^{17}$ N. Rupp,${ }^{11}$ R. Saldanha, ${ }^{19}$ J. M. F. dos Santos, ${ }^{6}$ G. Sartorelli, ${ }^{4}$ M. Scheibelhut, ${ }^{5}$ S. Schindler, ${ }^{5}$ J. Schreiner ${ }^{11}$ M. Schumann, ${ }^{13}$ L. Scotto Lavina, ${ }^{21}$ M. Selvi, ${ }^{4}$ P. Shagin, ${ }^{20}$ E. Shockley, ${ }^{19}$ M. Silva, ${ }^{6}$ H. Simgen, ${ }^{11}$ M. v. Sivers, ${ }^{13, \dagger}$ A. Stein ${ }^{22}$ S. Thapa, ${ }^{19}$ D. Thers, ${ }^{15}$ A. Tiseni,${ }^{2}$ G. Trinchero, ${ }^{18}$ C. Tunnell, ${ }^{19, \|}$ M. Vargas,${ }^{17}$ N. Upole, ${ }^{19}$ H. Wang, ${ }^{22}$ Z. Wang, ${ }^{3}$ Y. Wei, ${ }^{8}$ C. Weinheimer, ${ }^{17}$ J. Wulf, ${ }^{8}$ J. Ye, ${ }^{16}$ Y. Zhang, ${ }^{1}$ and T. Zhu ${ }^{1}$

\section{(XENON Collaboration)}

\author{
${ }^{1}$ Physics Department, Columbia University, New York, New York 10027, USA \\ ${ }^{2}$ Nikhef and the University of Amsterdam, Science Park, 1098XG Amsterdam, Netherlands \\ ${ }^{3}$ INFN-Laboratori Nazionali del Gran Sasso and Gran Sasso Science Institute, 67100 L'Aquila, Italy \\ ${ }^{4}$ Department of Physics and Astrophysics, University of Bologna and INFN-Bologna, 40126 Bologna, Italy \\ ${ }^{5}$ Institut für Physik and Exzellenzcluster PRISMA, Johannes Gutenberg-Universität Mainz, 55099 Mainz, Germany \\ ${ }^{6}$ LIBPhys, Department of Physics, University of Coimbra, 3004-516 Coimbra, Portugal \\ ${ }^{7}$ New York University Abu Dhabi, Abu Dhabi, United Arab Emirates \\ ${ }^{8}$ Physik-Institut, University of Zurich, 8057 Zurich, Switzerland \\ ${ }^{9}$ Oskar Klein Centre, Department of Physics, Stockholm University, AlbaNova, Stockholm SE-10691, Sweden \\ ${ }^{10}$ Department of Physics, Applied Physics and Astronomy, Rensselaer Polytechnic Institute, Troy, New York 12180, USA \\ ${ }^{11}$ Max-Planck-Institut für Kernphysik, 69117 Heidelberg, Germany \\ ${ }^{12}$ Department of Particle Physics and Astrophysics, Weizmann Institute of Science, Rehovot 7610001, Israel \\ ${ }^{13}$ Physikalisches Institut, Universität Freiburg, 79104 Freiburg, Germany \\ ${ }^{14}$ Department of Physics and Astronomy, Purdue University, West Lafayette, Indiana 47907, USA \\ ${ }^{15}$ SUBATECH, IMT Atlantique, CNRS/IN2P3, Université de Nantes, Nantes 44307, France \\ ${ }^{16}$ Department of Physics, University of California, San Diego, California 92093, USA \\ ${ }^{17}$ Institut für Kernphysik, Westfälische Wilhelms-Universität Münster, 48149 Münster, Germany \\ ${ }^{18}$ INFN-Torino and Osservatorio Astrofisico di Torino, 10125 Torino, Italy \\ ${ }^{19}$ Department of Physics and Kavli Institute for Cosmological Physics, University of Chicago, Chicago, Illinois 60637, USA \\ ${ }^{20}$ Department of Physics and Astronomy, Rice University, Houston, Texas 77005, USA \\ ${ }^{21}$ LPNHE, Université Pierre et Marie Curie, Université Paris Diderot, CNRS/IN2P3, Paris 75252, France \\ ${ }^{22}$ Physics \& Astronomy Department, University of California, Los Angeles, California 90095, USA \\ (Received 25 May 2017; revised manuscript received 2 August 2017; published 30 October 2017)
}

We report the first dark matter search results from XENON1T, a 2000-kg-target-mass dual-phase (liquid-gas) xenon time projection chamber in operation at the Laboratori Nazionali del Gran Sasso in Italy and the first ton-scale detector of this kind. The blinded search used 34.2 live days of data acquired between November 2016 and January 2017. Inside the (1042 \pm 12$)$-kg fiducial mass and in the [5,40] $\mathrm{keV}_{\mathrm{nr}}$ energy range of interest for weakly interacting massive particle (WIMP) dark matter searches, the electronic recoil background was $(1.93 \pm 0.25) \times 10^{-4}$ events $/\left(\mathrm{kg} \times\right.$ day $\left.\times \mathrm{keV}_{\mathrm{ee}}\right)$, the lowest ever achieved in such a dark matter detector. A profile likelihood analysis shows that the data are consistent with the background-only

Published by the American Physical Society under the terms of the Creative Commons Attribution 4.0 International license. Further distribution of this work must maintain attribution to the author(s) and the published article's title, journal citation, and DOI. 
hypothesis. We derive the most stringent exclusion limits on the spin-independent WIMP-nucleon interaction cross section for WIMP masses above $10 \mathrm{GeV} / c^{2}$, with a minimum of $7.7 \times 10^{-47} \mathrm{~cm}^{2}$ for $35-\mathrm{GeV} / c^{2}$ WIMPs at $90 \%$ C.L.

DOI: 10.1103/PhysRevLett.119.181301

Modern cosmology precisely describes observational data from the galactic to the cosmological scale with the $\Lambda$ cold dark matter model [1,2]. This model requires a nonrelativistic nonbaryonic component of the Universe called dark matter, with an energy density of $\Omega_{c} h^{2}=$ $0.1197 \pm 0.0022$ as measured by Planck [3]. Theories beyond the standard model of particle physics (e.g., supersymmetry [4]) often attribute this energy density to weakly interacting massive particles (WIMPs) that may be detectable by underground detectors $[5,6]$.

The XENON1T experiment is designed primarily for detecting nuclear recoils (NRs) from WIMP-nucleus scattering, continuing the XENON program $[7,8]$ that employs dual-phase (liquid-gas) xenon time projection chambers (TPCs) [8,9]. With a total mass of $\sim 3200 \mathrm{~kg}$ of ultrapure liquid xenon-more than 2 orders of magnitude larger than the initial detector of the XENON project [7]-XENON1T is the first detector of such scale realized to date. It is located at the Laboratori Nazionali del Gran Sasso in Italy, at an average depth of $3600 \mathrm{~m}$ water equivalent. The approximately $97-\mathrm{cm}-$ long by $96-\mathrm{cm}$-wide cylindrical TPC encloses $(2004 \pm 5) \mathrm{kg}$ of liquid xenon (LXe), while another $\sim 1200 \mathrm{~kg}$ provides additional shielding. The TPC is mounted at the center of a 9.6-m-diameter, 10-m-tall water tank to shield it from ambient radioactivity. An adjacent service building houses the xenon storage, cryogenics plant, data acquisition, and slow control system. The water tank is mounted with 84 photomultiplier tubes (PMTs) as part of a Cherenkov muon veto [10]. The TPC is instrumented with 248 3-in. Hamamatsu R11410-21 PMTs arranged in two arrays above and below the LXe target $[11,12]$. Interactions in the target produce scintillation photons (S1) and ionization electrons. The electrons drift in a $(116.7 \pm 7.5) \mathrm{V} / \mathrm{cm}$ electric field towards the liquid-gas interface at the top of the TPC. They are extracted into the gas by an electric field $E_{\text {gas }}>10 \mathrm{kV} / \mathrm{cm}$ where, via electroluminescence, they produce a proportional scintillation signal (S2). This charge-to-light amplification allows for the detection of single electrons [13,14]. The ratio of the $S 2$ to $S 1$ signals is determined by both the ratio of ionization to excitation in the initial interaction and subsequent partial recombination of the ionization, with lower $S 2 / S 1$ for NR signals than electronic recoils (ERs) from $\gamma$ and $\beta$ radiation.

Here, we report on 34.2 live days of blinded dark matter search data from the first science run of the experiment. The run started on November 22, 2016, and ended on January 18, 2017, when an earthquake temporarily interrupted detector operations. The detector's temperature, pressure, and liquid level remained stable at $(177.08 \pm 0.04) \mathrm{K}$,
$(1.934 \pm 0.001)$ bar, and $(2.5 \pm 0.2) \mathrm{mm}$ respectively, where the liquid level was measured above the grounded electrode separating the drift and extraction field regions. While the PMT high voltage remained stable during the run, 27 PMTs were turned off for the dark matter search and 8 were masked in the analysis due to low single-photoelectron (PE) detection efficiency. The PMT response was calibrated periodically using pulsed light-emitting diode data [15]. The xenon was continuously purified in the gas phase through hot metal getters, leading to an increase in the electron lifetime from 350 to $500 \mu \mathrm{s}$, with an average of $452 \mu \mathrm{s} ; 673 \mu \mathrm{s}$ is the drift time over the length of the TPC. Using cryogenic distillation [16], the ${ }^{\text {nat }} \mathrm{Kr}$ concentration in the LXe was reduced while the TPC was in operation, from $(2.60 \pm 0.05) \mathrm{ppt}(\mathrm{mol} / \mathrm{mol})$ at the beginning of the science run to $(0.36 \pm 0.06) \mathrm{ppt}$ one month after the end of the science run, as measured by rare-gas mass spectrometry [17] on samples extracted from the detector. The ${ }^{214} \mathrm{~Pb}$ event rate was $(0.8-1.9) \times 10^{-4}$ events $/\left(\mathrm{kg} \times\right.$ day $\left.\times \mathrm{keV}_{\mathrm{ee}}\right)$ in the low-energy range of interest for WIMP searches, where the bounds are set using in situ $\alpha$ spectroscopy on ${ }^{218} \mathrm{Po}$ and ${ }^{214} \mathrm{Po}$. The ${ }^{222} \mathrm{Rn}$ concentration was reduced by $\sim 20 \%$ relative to the equilibrium value using the krypton distillation column in inverse mode [18].

The data acquisition (DAQ) system continuously recorded individual PMT signals. The efficiency for recording single-PE pulses was $92 \%$ on average during the science run, and stable to within $2 \%$. A software trigger analyzed the PMT pulses in real time, allowing for continuous monitoring of the PMTs. The trigger detected S2s larger than 200 PE with $99 \%$ efficiency, and saved $1 \mathrm{~ms}$ before and after these to ensure that small $S 1$ s were captured. An analog-sum waveform was separately digitized together with a signal recording when any of the digitizers were inhibited. The average DAQ live time was $92 \%$ during the science run.

Physical signals are reconstructed from raw data by finding photon hits in each PMT channel, then clustering and classifying groups of hits as $S 1$ or $S 2$ using the PAX software. For $S 1 \mathrm{~s}$, we require that hits from three or more PMTs occur within $50 \mathrm{~ns}$. To tune the signal reconstruction algorithms and compute their efficiency for detecting NRs - shown in blue in Fig. 1-we used a Monte Carlo code that reproduces the shapes of $S 1$ s and $S 2$ s as determined by the interaction physics, light propagation, and detector-electronics chain. This was validated against ${ }^{83 m} \mathrm{Kr}$ and ${ }^{220} \mathrm{Rn}$ calibration data.

The interaction position is reconstructed from the toparray PMT hit pattern of the $S 2$ (for the transverse position) and the time difference between $S 1$ and $S 2$ (for depth). The 


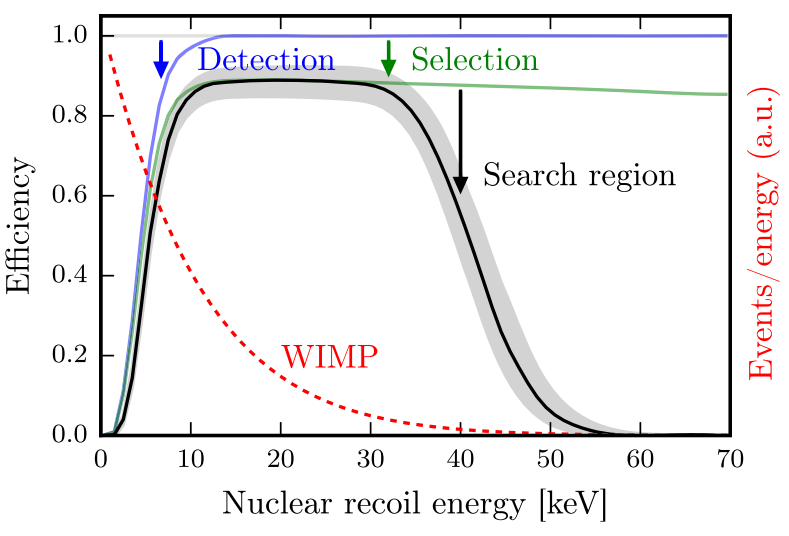

FIG. 1. NR detection efficiency in the fiducial mass at successive analysis stages as a function of recoil energy. At low energy, the detection efficiency (blue line) dominates. At $20 \mathrm{keV}$, the efficiency is $82 \%$, primarily due to event selection losses (green line). At high energies, the effect of restricting our data to the search region described in the text (black line) is dominant. The black line is our final NR efficiency, with uncertainties shown in gray. The NR energy spectrum shape of a $50-\mathrm{GeV} / c^{2}$ WIMP (in a.u.) is shown in red for reference.

$S 2$ transverse position is given by maximizing a likelihood based on an optical simulation of the photons produced in the $S 2$ amplification region. The simulation-derived transverse resolution is $\sim 2 \mathrm{~cm}$ at our $S 2$ analysis threshold of $200 \mathrm{PE}$ (uncorrected). The interaction position is corrected for drift field nonuniformities derived from a finite element simulation, which is validated using ${ }^{83 m} \mathrm{Kr}$ calibration data. We correct $S 2$ s for electron losses during drift, and both $S 1$ s and $S 2$ s for spatial variations of up to $30 \%$ and $15 \%$, respectively, inferred from ${ }^{83 m} \mathrm{Kr}$ calibration data. These spatial variations are mostly due to geometric light-collection effects. The resulting corrected quantities are called $c S 1$ and $c S 2$. As the bottom PMT array has a more homogeneous response to $S 2$ light than the top, this analysis uses $c S 2_{b}$, a quantity similar to $c S 2$ based on the $S 2$ signal seen only by the bottom PMTs.

To calibrate XENON1T, we acquired 3.0 days of data with ${ }^{220} \mathrm{Rn}$ injected into the LXe (for low-energy ERs), 3.3 days with ${ }^{83 m} \mathrm{Kr}$ injected into the LXe (for the spatial response) and 16.3 days with an external ${ }^{241} \mathrm{AmBe}$ source (for low-energy NRs). The data from the ${ }^{220} \mathrm{Rn}$ [19] and ${ }^{241} \mathrm{AmBe}$ calibrations are shown in Figs. 2(a) and 2(b), respectively. Following the method described in Ref. [20] with a $W$ value of $13.7 \mathrm{eV}$, we extracted the photon gain $g_{1}=(0.144 \pm 0.007) \mathrm{PE}$ per photon and the electron gain $g_{2}=(11.5 \pm 0.8) \mathrm{PE}$ (in the bottom array, 2.86 times lower than if both arrays are used) per electron in the fiducial mass by fitting the anticorrelation of $c S 2_{b}$ and $c S 1$ for signals with known energy from ${ }^{83 m} \mathrm{Kr}(41.5 \mathrm{keV}),{ }^{60} \mathrm{Co}$ from detector materials (1.173 and $1.332 \mathrm{MeV})$, and from decays of metastable ${ }^{131 m} \mathrm{Xe}(164 \mathrm{keV})$ and ${ }^{129 m} \mathrm{Xe}$ $(236 \mathrm{keV})$ produced during the ${ }^{241} \mathrm{AmBe}$ calibration. The

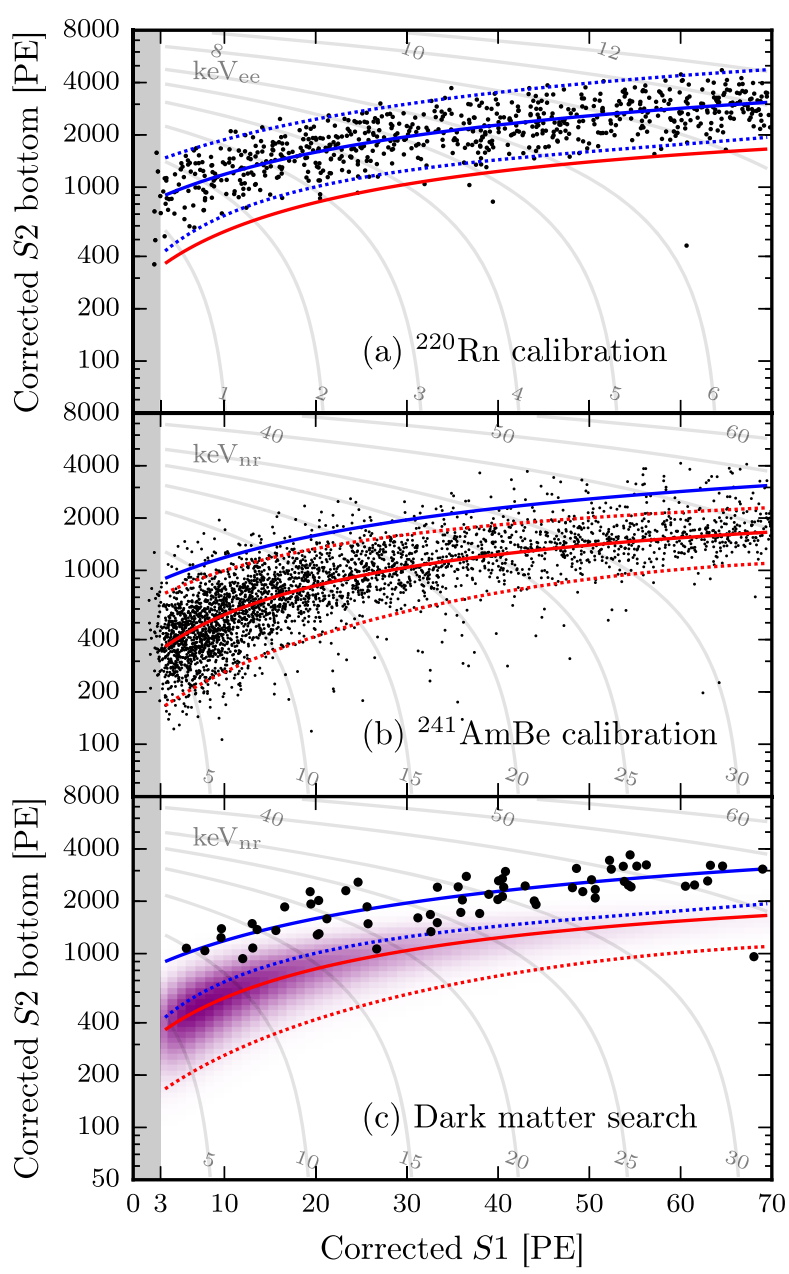

FIG. 2. Observed data in $c S 2_{b}$ vs $c S 1$ for (a) ${ }^{220} \mathrm{Rn}$ ER calibration, (b) ${ }^{241} \mathrm{AmBe} \mathrm{NR}$ calibration, and (c) the 34.2-day dark matter search. Solid and dotted lines indicate the median and $\pm 2 \sigma$ quantiles, respectively, of simulated event distributions (with the simulation fitted to calibration data). Red lines show $\mathrm{NR}$ (fitted to ${ }^{241} \mathrm{AmBe}$ ) and blue ER (fitted to ${ }^{220} \mathrm{Rn}$ ). In (c), the purple distribution indicates the signal model of a $50-\mathrm{GeV} / c^{2}$ WIMP. Thin gray lines and labels indicate contours of the constant combined energy scale in $\mathrm{keV}$ for (a) ER and (b),(c) NR. Data below $c S 1=3 \mathrm{PE}$ (the gray region) are not in our analysis region of interest and are shown only for completeness.

$c S 1$ and $c S 2_{b}$ yields are stable in time within $0.77 \%$ and $1.2 \%$, respectively, as determined by the ${ }^{83 m} \mathrm{Kr}$ calibrations.

WIMPs are expected to induce low-energy single-scatter NRs. Events that are not single scatters in the LXe are removed by several event-selection cuts: (1) a single $S 2$ above 200 PE must be present and any other $S 2$ s must be compatible with single electrons from photoionization of impurities or delayed extraction; (2) an event must not closely follow a high-energy event (e.g., within $8 \mathrm{~ms}$ after a $3 \times 10^{5} \mathrm{PE} \mathrm{S2}$ ), which can cause long tails of single electrons; (3) the $S 2$ signal's duration must be consistent with the depth of the interaction as inferred from the drift time; (4) the $S 1$ and $S 2$ hit patterns must be consistent with 
the reconstructed position at which these signals were produced; (5) no more than $300 \mathrm{PE}$ of uncorrelated single electrons and PMT dark counts must appear in the region before the $S 2$. Single-scatter NR events within the $[5,40] \mathrm{keV}_{\mathrm{nr}}$ energy range pass these selections with $>82 \%$ probability, as determined using simulated events or control samples derived from calibration, and shown in green in Fig. 1.

The dark matter search uses a cylindrical (1042 \pm 12)-kg fiducial mass, which was defined before unblinding using the reconstructed spatial distribution of ERs in the dark matter search data and the energy distribution of ERs from ${ }^{220} \mathrm{Rn}$. We restrict the search to $c S 1 \in[3,70] \mathrm{PE}$ and $c S 2_{b} \in[50,8000] \mathrm{PE}$, which causes little additional loss of WIMP signals, as shown in black in Fig. 1.

Table I lists the six sources of background we consider inside the fiducial mass and inside the search region. For illustration, we also list the expected rate in a reference region between the NR median and the $-2 \sigma$ quantile in $c S 2_{b}$ [i.e., between the red lines in Fig. 2(c)], for which Fig. 3 shows the background model projected onto $c S 1$. This reference region would contain about half of the WIMP candidate events, while excluding $99.6 \%$ of the ER background. The WIMP search likelihood analysis uses the full search region. Below, we describe each background component in more detail: all event rates are understood to be inside the fiducial mass and the full search region.

First, our background model includes ERs, primarily from $\beta$ decays of ${ }^{85} \mathrm{Kr}$ and the intrinsic ${ }^{222} \mathrm{Rn}$-progeny ${ }^{214} \mathrm{~Pb}$, which cause a flat energy spectrum in the energy range of interest [9]. The ER background model is based on a simulation of the detector response. We use a model similar to that in Ref. [21] to convert the energy deposition from ERs into scintillation photons and ionization electrons, which we fit to ${ }^{220} \mathrm{Rn}$ calibration data in $\left(c S 1, c S 2_{b}\right)$ space [Fig. 2(a)].

The best-fit photon yield and recombination fluctuations are comparable to those of Ref. [21]. The model accounts

TABLE I. Expected number of events for each background component in the fiducial mass; in the full $c S 1 \in[3,70] \mathrm{PE}$, $c S 2_{b} \in[50,8000] \mathrm{PE}$ search region and in a reference region between the NR median and the $-2 \sigma$ quantile in $c S 2_{b}$. Uncertainties $<0.005$ events are omitted. The ER rate is unconstrained in the likelihood; for illustration, we list the best-fit values to the data in parentheses.

\begin{tabular}{lll}
\hline \hline & \multicolumn{1}{c}{ Full } & \multicolumn{1}{c}{ Reference } \\
\hline Electronic recoils (ERs) & $(62 \pm 8)$ & $\left(0.26_{-0.07}^{+0.11}\right)$ \\
Radiogenic neutrons $(n)$ & $0.05 \pm 0.01$ & 0.02 \\
CNNS $(\nu)$ & 0.02 & 0.01 \\
Accidental coincidences (acc) & $0.22 \pm 0.01$ & 0.06 \\
Wall leakage (wall) & $0.5 \pm 0.3$ & 0.01 \\
Anomalous (anom) & $0.10_{-0.07}^{+0.10}$ & $0.01 \pm 0.01$ \\
Total background & $63 \pm 8$ & $0.36_{-0.07}^{+0.11}$ \\
50-GeV $/ c^{2}, 10^{-46}-\mathrm{cm}^{2}$ WIMP & $1.66 \pm 0.01$ & $0.82 \pm 0.06$ \\
\hline \hline
\end{tabular}

for uncertainties of $g_{1}$ and $g_{2}$, spatial variations of the $S 1$ and $S 2$ light-collection efficiencies, the electron-extraction efficiency, reconstruction and event-selection efficiency, and time dependence of the electron lifetime. The rate of ERs is not constrained in the likelihood analysis, even though we have independent concentration measurements for ${ }^{214} \mathrm{~Pb}$ and ${ }^{85} \mathrm{Kr}$, since the most stringent constraint comes from the search data themselves.

Second and third, our background model includes two sources of NRs: radiogenic neutrons contribute $(0.05 \pm 0.01)$ events and coherent neutrino-nucleus scattering (CNNS) $\sim 0.02$ events. Cosmogenically produced neutrons are estimated to contribute $O\left(10^{-3}\right)$ events even without muon-veto tagging. The NR background model is built from a detector response simulation that shares the same detector parameters and associated systematic uncertainties as the ER background model above. The main difference is the energy-conversion model, where we use the model and parametrization from NEST [22]. We obtain the XENON1T response to NRs by fitting the ${ }^{241} \mathrm{AmBe}$ calibration data [Fig. 2(b)] with the light and charge yields from Ref. [22] as priors. Our NR response model is therefore constrained by the global fit of external data. It is also used to predict the WIMP signal models in $\left(c S 1, c S 2_{b}\right)$ space. The $S 1$ detection efficiency, which is responsible for our lowenergy threshold, is consistent with its prior $(0.7 \sigma)$.

Fourth, accidental coincidences of uncorrelated $S 1 \mathrm{~s}$ and $S 2$ s are expected to contribute $(0.22 \pm 0.01)$ background events. We estimated their rate and $\left(c S 1, c S 2_{b}\right)$ distribution using isolated $S 1$ and $S 2$ signals, which are observed to be at $(0.78 \pm 0.01) \mathrm{Hz}$ and $(3.23 \pm 0.03) \mathrm{mHz}$, respectively, before applying $S 2$ selections. The effect of our event selection on the accidental coincidence rate is included,

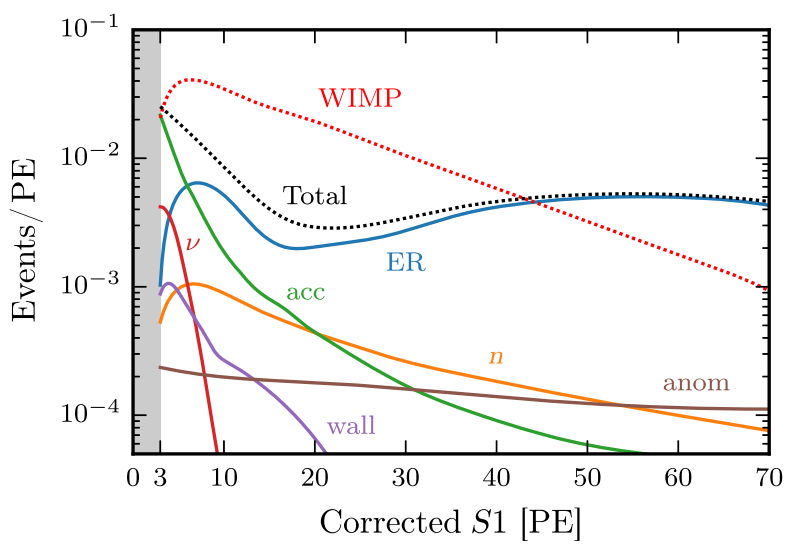

FIG. 3. Background model in the fiducial mass in a reference region between the NR median and the $-2 \sigma$ quantile in $c S 2_{b}$, projected onto $c S 1$. Solid lines show that the expected number of events from individual components listed in Table I; the labels match the abbreviations shown in the table. The dotted black line "Total" shows the total background model, while the dotted red line "WIMP" shows an $m=50 \mathrm{GeV} / c^{2}, \sigma=10^{-46} \mathrm{~cm}^{2}$ WIMP signal for comparison. 
similar to Ref. [23]. Isolated $S 1$ s may arise from interactions in regions of the detector with poor charge collection, such as below the cathode, suppressing an associated $c S 2$ signal. Isolated $S 2 \mathrm{~s}$ might arise from photoionization at the electrodes, from regions with poor light collection, or from delayed extraction [24]. Most accidental events are expected at low $c S 1$ and at lower $c S 2_{b}$ than at typical NRs.

Fifth, inward-reconstructed events from near the TPC's polytetrafluoroethylene wall are expected to contribute $(0.5 \pm 0.3)$ events, with the rate and $\left(c S 1, c S 2_{b}\right)$ spectrum extrapolated from events outside the fiducial mass. Most of these events would appear at unusually low $c S 2_{b}$ due to charge losses near the wall. The inward reconstruction is due to limited position reconstruction resolution, limited especially for small $S 2$ s, near the 5 (out of 36) top PMTs in the outermost ring that are unavailable in this analysis.

Sixth and last, we add a small uniform background in the $\left(c S 1, \log c S 2_{b}\right)$ space for ER events with an anomalous $c S 2{ }_{b}$. Such anomalous leakage beyond accidental coincidences was observed in XENON100 [23], and one such event is seen in the ${ }^{220} \mathrm{Rn}$ calibration data [Fig. 2(a)]. If these were not ${ }^{220} \mathrm{Rn}$-induced events, their rate would scale with exposure and we would see numerous such events in the WIMP search data. We do not observe this and therefore assume their rate is proportional to the ER rate, at $0.10_{-0.07}^{+0.10}$ events based on the outliers observed in the ${ }^{220} \mathrm{Rn}$ calibration data. The physical origin of these events is under investigation.

The WIMP search data in a predefined signal box were blinded (99\% of ERs were accessible) until the event selection and the fiducial mass boundaries were finalized. We performed a staged unblinding, starting with an exposure of four live days distributed evenly throughout the search period. No changes to either the event-selection or background types were made at any stage.

A total of 63 events in the 34.2-day dark matter search data pass the selection criteria and are within the $c S 1 \in$ $[3,70] \mathrm{PE}, c S 2_{b} \in[50,8000] \mathrm{PE}$ search region used in the likelihood analysis [Fig. 2(c)]. None are within $10 \mathrm{~ms}$ of a muon-veto trigger. The data are compatible with the ER energy spectrum in Ref. [9] and implies an ER rate of $(1.93 \pm 0.25) \times 10^{-4}$ events $/\left(\mathrm{kg} \times\right.$ day $\left.\times \mathrm{keV}_{\text {ee }}\right)$, compatible with our prediction of $(2.3 \pm 0.2) \times 10^{-4}$ events/ $\left(\mathrm{kg} \times\right.$ day $\left.\times \mathrm{keV}_{\mathrm{ee}}\right)[9]$ updated with the lower $\mathrm{Kr}$ concentration measure in the current science run. This is the lowest ER background ever achieved in such a dark matter experiment. A single event far from the bulk distribution was observed at $c S 1=68.0 \mathrm{PE}$ in the initial 4-day unblinding stage. This appears to be a bona fide event, though its location in $\left(c S 1, c S 2_{b}\right)$ [see Fig. 2(c)] is extreme for all WIMP signal models and background models other than anomalous leakage and accidental coincidence. One event at $c S 1=26.7 \mathrm{PE}$ is at the $-2.4 \sigma$ ER quantile.

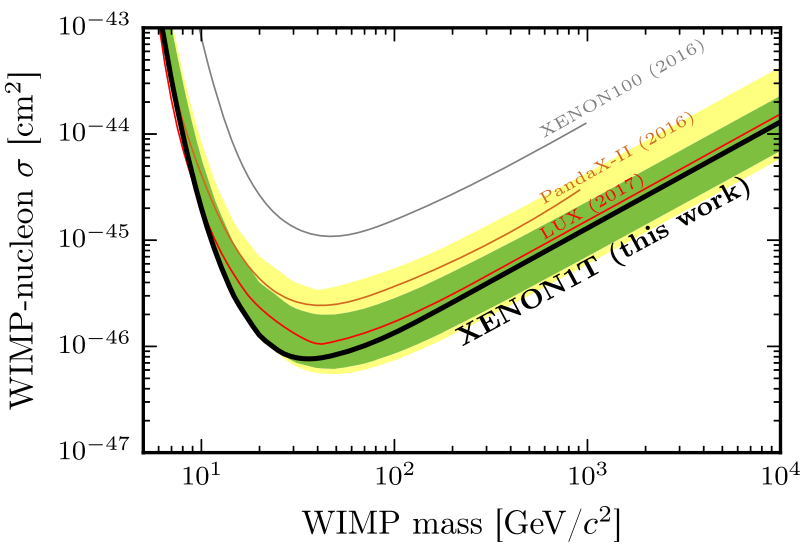

FIG. 4. The spin-independent WIMP-nucleon cross section limits as a function of the WIMP mass at $90 \%$ confidence level (black line) for this run of XENON1T. In green and yellow are the $1 \sigma$ and $2 \sigma$ sensitivity bands. Results from LUX [27] (the red line), PandaX-II [28] (the brown line), and XENON100 [23] (the gray line) are shown for reference.

For the statistical interpretation of the results, we use an extended unbinned profile likelihood test statistic in $(c S 1$, $c S 2_{b}$ ). We propagate the uncertainties on the most significant shape parameters (two for NR, two for ER) inferred from the posteriors of the calibration fits to the likelihood. The uncertainties on the rate of each background component mentioned above are also included. The likelihood ratio distribution is approximated by its asymptotic distribution [25]; preliminary toy Monte Carlo checks show that the effect on the exclusion significance of this conventional approximation is well within the result's statistical and systematic uncertainties. To account for mismodeling of the ER background, we also calculated the limit using the procedure in Ref. [26], which yields a similar result.

The data are consistent with the background-only hypothesis. Figure 4 shows the $90 \%$ confidence level upper limit on the spin-independent WIMP-nucleon cross section, power constrained at the $-1 \sigma$ level of the sensitivity band [29]. The final limit is within $10 \%$ of the unconstrained limit for all WIMP masses. For the WIMP energy spectrum, we assume a standard isothermal WIMP halo with $v_{0}=220 \mathrm{~km} / \mathrm{s}$, $\rho_{\mathrm{DM}}=0.3 \mathrm{GeV} / \mathrm{cm}^{3}, \quad v_{\mathrm{esc}}=544 \mathrm{~km} / \mathrm{s}$, and the Helm form factor for the nuclear cross section [30]. No light or charge emission is assumed for WIMPs below 1-keV recoil energy. For all WIMP masses, the background-only hypothesis provides the best fit, with none of the nuisance parameters representing the uncertainties discussed above deviating appreciably from their nominal values. Our results improve upon the previously strongest spinindependent WIMP limit for masses above $10 \mathrm{GeV} / c^{2}$. Our strongest exclusion limit is for $35-\mathrm{GeV} / c^{2}$ WIMPs, at $7.7 \times 10^{-47} \mathrm{~cm}^{2}$.

These first results demonstrate that XENON1T has the lowest low-energy background level ever achieved by a 
dark matter experiment. The sensitivity of XENON1T is the best to date above $20 \mathrm{GeV} / c^{2}$, up to twice the LUX sensitivity above $100 \mathrm{GeV} / c^{2}$, and continues to improve with more data. The experiment resumed operation shortly after the January 18, 2017, earthquake and continues to record data.

We gratefully acknowledge support from the National Science Foundation, the Swiss National Science Foundation, the German Ministry for Education and Research, Max Planck Gesellschaft, Deutsche Forschungsgemeinschaft, the Netherlands Organisation for Scientific Research (NWO), NLeSC, Weizmann Institute of Science, I-CORE, Pazy-Vatat, Initial Training Network Invisibles (Marie Curie Actions, PITNGA-2011-289442), Fundacao para a Ciencia e a Tecnologia, Region des Pays de la Loire, Knut and Alice Wallenberg Foundation, Kavli Foundation, and Instituto Nazionale di Fisica Nucleare. J. Conrad received support from a Wallenberg Academy Fellowship. Data processing was performed using infrastructures from the Open Science Grid and the European Grid Initiative. We are grateful to Laboratori Nazionali del Gran Sasso for hosting and supporting the XENON project.

*jaalbers@ nikhef.nl

${ }^{\dagger}$ Also at Albert Einstein Center for Fundamental Physics, University of Bern, Bern, Switzerland.

${ }^{*}$ Also at Coimbra Engineering Institute, Coimbra, Portugal.

§uillaume.plante@astro.columbia.edu

tunnell@uchicago.edu

ॠxenon@lngs.infn.it

[1] S. Dodelson, Modern Cosmology (Academic Press, Amsterdam, 2003).

[2] E. W. Kolb and M. S. Turner, The early Universe, Front. Phys. 69, 1 (1990).

[3] P. A. R. Ade et al. (Planck Collaboration), Planck 2015 results. XIII. Cosmological parameters, Astron. Astrophys. 594, A13 (2016).

[4] G. Jungman, M. Kamionkowski, and K. Griest, Supersymmetric dark matter, Phys. Rep. 267, 195 (1996).

[5] M. W. Goodman and E. Witten, Detectability of certain dark-matter candidates, Phys. Rev. D 31, 3059 (1985).

[6] T. Marrodán Undagoitia and L. Rauch, Dark matter directdetection experiments, J. Phys. G 43, 013001 (2016).

[7] E. Aprile et al. (XENON Collaboration), Design and performance of the XENON10 dark matter experiment, Astropart. Phys. 34, 679 (2011).

[8] E. Aprile et al. (XENON100 Collaboration), The XENON100 dark matter experiment, Astropart. Phys. 35, 573 (2012).

[9] E. Aprile et al. (XENON Collaboration), Physics reach of the XENON1T dark matter experiment, J. Cosmol. Astropart. Phys. 04 (2016) 027.

[10] E. Aprile et al. (XENON1T Collaboration), Conceptual design and simulation of a water Cherenkov muon veto for the XENON1T experiment, J. Instrum. 9, P11006 (2014).
[11] E. Aprile et al. (XENON Collaboration), Lowering the radioactivity of the photomultiplier tubes for the XENON1T dark matter experiment, Eur. Phys. J. C 75, 546 (2015).

[12] P. Barrow et al., Qualification tests of the R11410-21 photomultiplier tubes for the XENON1T detector, J. Instrum. 12, P01024 (2017).

[13] B. Edwards et al. (ZEPLIN-II Collaboration), Measurement of single electron emission in two-phase xenon, Astropart. Phys. 30, 54 (2008).

[14] E. Aprile et al. (XENON100 Collaboration), Observation and applications of single-electron charge signals in the XENON100 experiment, J. Phys. G 41, 035201 (2014).

[15] R. Saldanha, L. Grandi, Y. Guardincerri, and T. Wester, Model independent approach to the single photoelectron calibration of photomultiplier tubes, Nucl. Instrum. Methods Phys. Res., Sect. A 863, 35 (2017).

[16] E. Aprile et al. (XENON Collaboration), Removing krypton from xenon by cryogenic distillation to the $p p q$ level, Eur. Phys. J. C 77, 275 (2017).

[17] S. Lindemann and H. Simgen, Krypton assay in xenon at the $p p q$ level using a gas chromatographic system and mass spectrometer, Eur. Phys. J. C 74, 2746 (2014).

[18] E. Aprile et al. (XENON Collaboration), Online ${ }^{222} \mathrm{Rn}$ removal by cryogenic distillation in the XENON100 experiment, Eur. Phys. J. C 77, 358 (2017).

[19] E. Aprile et al. (XENON Collaboration), Results from a calibration of XENON100 using a source of dissolved radon-220, Phys. Rev. D 95, 072008 (2017).

[20] C. E. Dahl, Ph.D. thesis, Princeton University, 2009.

[21] D. S. Akerib et al. (LUX Collaboration), Tritium calibration of the LUX dark matter experiment, Phys. Rev. D 93, 072009 (2016).

[22] B. Lenardo, K. Kazkaz, A. Manalaysay, J. Mock, M. Szydagis, and M. Tripathi, A global analysis of light and charge yields in liquid xenon, IEEE Trans. Nucl. Sci. 62, 3387 (2015).

[23] E. Aprile et al. (XENON Collaboration), XENON100 dark matter results from a combination of 477 live days, Phys. Rev. D 94, 122001 (2016).

[24] P. Sorensen, Electron train backgrounds in liquid xenon dark matter search detectors are indeed due to thermalization and trapping, arXiv:1702.04805.

[25] G. Cowan, K. Cranmer, E. Gross, and O. Vitells, Asymptotic formulae for likelihood-based tests of new physics, Eur. Phys. J. C 71, 1554 (2011); ErratumEur. Phys. J. C73, 2501(E) (2013).

[26] N. Priel, L. Rauch, H. Landsman, A. Manfredini, and R. Budnik, A model independent safeguard for unbinned likelihood, J. Cosmol. Astropart. Phys. 05 (2017) 013.

[27] D. S. Akerib et al. (LUX Collaboration), Results from a Search for Dark Matter in the Complete LUX Exposure, Phys. Rev. Lett. 118, 021303 (2017).

[28] A. Tan et al. (PandaX-II Collaboration), Dark Matter Results from First 98.7-day Data of PandaX-II Experiment, Phys. Rev. Lett. 117, 121303 (2016).

[29] G. Cowan, K. Cranmer, E. Gross, and O. Vitells, Powerconstrained limits, arXiv:1105.3166.

[30] J. Lewin and P. Smith, Review of mathematics, numerical factors, and corrections for dark matter experiments based on elastic nuclear recoil, Astropart. Phys. 6, 87 (1996). 